\title{
Positive polynomials on fibre products
}

\author{
Salma Kuhlmann ${ }^{\text {a }}$, Mihai Putinar ${ }^{b}$ \\ a Department of Mathematics and Statistics, University of Saskatchewan, S7N 5E6, Canada \\ $\mathrm{b}^{\mathrm{b}}$ Mathematics Department, University of California, Santa Barbara, CA 93106, USA
}

\begin{abstract}
Recent investigations in optimization theory concerning the structure of positive polynomials with a sparsity pattern are interpreted in the more invariant language of (iterated) fibre products of real algebraic varieties. This opens the perspective of treating on a unifying basis the cases of positivity on unbounded supports, on non-semialgebraic supports, or of polynomials depending on countably many variables.
\end{abstract}

\section{Résumé}

Polynômes positifs sur des produits fibrés. Nous présentons une interprétation algébrique (dans le langage des produits fibrés de variétés algébriques) de résultats récents en théorie de l'optimisation concernant la structure de polynômes positifs (sur un sous ensemble compact et semi-algébrique $K \subset \mathbb{R}^{n}$ ) qui satisfont certaines conditions de séparation des variables dans leurs monômes. Ceci offre la perspective d'un traitement uniforme de tels polynômes, positifs sur $K$ non-compact, ou non-semi-algébrique, ainsi que pour des polynômes en un nombre dénombrable de variables.

\section{Introduction}

Let $A$ be a commutative ring with 1 . For simplicity we assume that $\mathbb{Q} \subset A$. A quadratic module $Q \subset A$ is a subset of $A$ such that $Q+Q \subset Q, 1 \in Q$ and $a^{2} Q \subset A$ for all $a \in A$. We denote by $Q(M ; A)$ the quadratic module generated in $A$ by the set $M$. That is $Q(M ; A)$ is the smallest subset of $A$ which is closed under addition and multiplication by squares $a^{2}, a \in A$, containing $M$ and the unit $1 \in A$. If $M$ is finite, we say that the quadratic module is finitely generated. A quadratic module which is also closed under multiplication is called a quadratic preordering.

In the terminology used throughout this Note, a real algebraic, affine variety $X \subset \mathbb{R}^{d}$ is the common zero set of a finite set of polynomials, and the algebra of regular functions on $X$ is

\footnotetext{
Partially supported by an NSERC Discovery Grant, Canada and the National Science Foundation-USA.

E-mail addresses: skuhlman@math.usask.ca (S. Kuhlmann), mputinar@math.ucsb.edu (M. Putinar).

URLs: http://www.math.usask.ca/ skuhlman (S. Kuhlmann), http://www.math.ucsb.edu/ mputinar (M. Putinar).
} 


$$
A=\mathbb{R}[X]=\mathbb{R}\left[x_{1}, \ldots, x_{d}\right] / I(X),
$$

where $I(X)$ is the radical ideal associated to $X$. The non-negativity set of a subset $S \subset \mathbb{R}[X]$ is

$$
P(S)=\{x \in X ; f(x) \geqslant 0, f \in S\} .
$$

The duality between finitely generated quadratic modules and non-negativity sets plays a similar role in semialgebraic geometry to the classical pairing between ideals and algebraic varieties, see [11] for details. A quadratic module $Q \subset \mathbb{R}[X]$ is archimedean if for every element $f \in \mathbb{R}[X]$ there exists a positive scalar $\alpha$ such that $1+\alpha f \in Q$. It is easy to see that the non-negativity set of an archimedean quadratic module is always compact.

The following Striktpositivestellensatz has attracted in the last decade a lot of attention from practitioners of polynomial optimization: Let $Q \subset \mathbb{R}\left[x_{1}, \ldots, x_{d}\right]$ be an archimedean quadratic module and assume that a polynomial $f$ is positive on $P(Q)$. Then $f \in Q$.

The above fact was discovered by the second author [12], generalizing Schmüdgen's Striktpositivestellensatz [14] for the finitely generated preordering associated to a compact non-negativity set.

The two main results of the present Note are Theorems 2.1 and 2.3. Theorem 2.1 generalizes the above Striktpositivstellensatz to certain fibre products of algebraic varieties, indexed over a rooted tree (see Section 2 below for this terminology). In the case of fibre products of affine spaces, we recover in Corollary 2.5 recently proved results on the representations of positive sparse polynomials (that is, polynomials in which every monomial involves only variables from blocks of variables satisfying certain overlap conditions); see [3,4,8,9,15]. It is worth remarking that Lasserre [8] uses in his proofs, as we do, a natural disintegration of measures argument, but this is done in a direct way, namely by relaxing the point evaluations in polynomial optimization over semialgebraic sets by averages over probability measures. The rooted tree projective system corresponds to what is known in the applied mathematics community as the running intersection property, see $[3,4,8]$. The benefits of Theorem 2.3 lie in the fact that non-semialgebraic supports can be described as projective limits of a sequence of semialgebraic ones, and also in the possibility of covering the case of polynomials depending on an infinity of variables.

Finally, the separation arguments in our proofs below seem to carry over to certain non-compact supports, for which a close form of the Striktpositivstellensatz is available (see for details $[6,7,10,13]$ ). In this case the quadratic modules $Q_{i}$ have to be replaced by the quadratic preorderings. We will resume this idea, with full details, in a forthcoming article.

\section{Main results}

Let $I$ be a non-empty set, endowed with a partial order relation $i \leqslant j$. A projective system of algebraic varieties indexed over $I$ consists of a family of varieties (affine in our case) $X_{i}, i \in I$, and morphisms $f_{i j}: X_{j} \rightarrow X_{i}$ defined whenever $i \leqslant j$, and satisfying the compatibility condition $f_{i k}=f_{i j} f_{j k}$ if $i \leqslant j \leqslant k$. The topological projective limit $X=$ proj.lim $\left(X_{i}, f_{i j}\right)$ is the universal object endowed with morphisms $f_{i}: X \rightarrow X_{i}$ satisfying the compatibility conditions $f_{i}=f_{i j} f_{j}, i \leqslant j$. See for instance [2] $\S 6$. A directed projective system carries the additional assumption on the index set that for every pair $i, j \in I$ there exists $k \in I$ satisfying $i \leqslant k$ and $j \leqslant k$.

A finite partially ordered set $I=\left\{i_{0}, \ldots, i_{n}\right\}$ is called a rooted tree if the order structure is generated by the inequalities $i_{1} \geqslant i_{0}$ and for every $k>1, i_{k} \geqslant i_{j(k)}$ with $j(k)<k$. Regarded as a graph, a rooted tree is obtained successively from the root $i_{0}$ by adding one branch at a time.

First we are concerned with finite projective systems of algebraic varieties:

Theorem 2.1. Let $\left(X_{i}, f_{i j}\right)$ be a finite projective system of real affine varieties, indexed by a rooted tree. Let $Q_{i} \subset$ $\mathbb{R}\left[X_{i}\right]$ be archimedean quadratic modules, subject to the coherence condition $f_{i j}^{*} Q_{i} \subseteq Q_{j}$. Let $p \in \sum_{i} f_{i}^{*} \mathbb{R}\left[X_{i}\right]$ be an element which is positive on the set $\bigcap_{i \in I} f_{i}^{-1} P\left(Q_{i}\right)$. Then $p \in \sum_{i} f_{i}^{*} Q_{i}$.

The proof consists in an iteration of the following scheme.

Let $Z=X_{1} \times_{Y} X_{2}$ be the fibre product of affine real varieties. Specifically

$$
f_{i}: X_{i} \longrightarrow Y, \quad i=1,2,
$$

are given morphisms and

$$
Z=\left\{\left(x_{1}, x_{2}\right) \in X_{1} \times X_{2} ; f_{1}\left(x_{1}\right)=f_{2}\left(x_{2}\right)\right\} .
$$


This is still an algebraic variety, with the ring of regular functions

$$
\mathbb{R}\left[X_{1} \times_{Y} X_{2}\right]=\mathbb{R}\left[X_{1}\right] \otimes_{\mathbb{R}[Y]} \mathbb{R}\left[X_{2}\right] .
$$

Denote by $u_{i}: Z \rightarrow X_{i}, i=1,2$, the projection maps, so that: $f_{1} u_{1}=f_{2} u_{2}$.

Lemma 2.2. With the above notation, let $Q_{i} \subset \mathbb{R}\left[X_{i}\right], i=1,2$, be Archimedean quadratic modules. If an element $p \in u_{1}^{*} \mathbb{R}\left[X_{1}\right]+u_{2}^{*} \mathbb{R}\left[X_{2}\right]$ is strictly positive on the set $u_{1}^{-1} P\left(Q_{1}\right) \cap u_{2}^{-1} P\left(Q_{2}\right)$, then $p \in u_{1}^{*} Q_{1}+u_{2}^{*} Q_{2}$.

The proof of the lemma follows an argument by contradiction, widely used in the functional analytic proofs of similar results. More precisely, assume that $p \notin u_{1}^{*} Q_{1}+u_{2}^{*} Q_{2}$. Since the two quadratic modules are achimedean, the element 1 (constant function) belongs to the algebraic interior of the convex cone $u_{1}^{*} Q_{1}+u_{2}^{*} Q_{2}$, regarded as a subset of the vector space $u_{1}^{*} \mathbb{R}\left[X_{1}\right]+u_{2}^{*} \mathbb{R}\left[X_{2}\right]$. In virtue of the Eidelheit-Kakutani separation theorem (see [5]), there exists a real linear functional

$$
L: u_{1}^{*} \mathbb{R}\left[X_{1}\right]+u_{2}^{*} \mathbb{R}\left[X_{2}\right] \longrightarrow \mathbb{R}
$$

satisfying

$$
L(p) \leqslant 0 \leqslant L(f), \quad f \in u_{1}^{*} Q_{1}+u_{2}^{*} Q_{2}, \quad L(1)>0 .
$$

By the archimedean assumption, there exists positive Borel measures $\mu_{i}$, compactly supported on $P\left(Q_{i}\right), i=1,2$, satisfying $\left(f_{1}\right)_{*} \mu_{1}=\left(f_{2}\right)_{*} \mu_{2}$ and such that

$$
L\left(u_{1}^{*} \phi_{1}+u_{2}^{*} \phi_{2}\right)=\int_{X_{1}} \phi_{1} \mathrm{~d} \mu_{1}+\int_{X_{2}} \phi_{2} \mathrm{~d} \mu_{2}, \quad \phi_{i} \in \mathbb{R}\left[X_{i}\right], i=1,2 .
$$

A classical disintegration of measures theorem (see [2] Proposition IX.2.13) yields a positive Borel measure $\mu$ on the space $Z$, satisfying $\left(u_{i}\right)_{*} \mu=\mu_{i}, i=1,2$ (provided the compatibility condition $\left(f_{1}\right)_{*} \mu_{1}=\left(f_{2}\right)_{*} \mu_{2}$ is fulfilled). Note that the measure $\mu$ representing the extension $\tilde{L}$ of $L$ to $\mathbb{R}[Z]$, is supported by the set $\Pi=u_{1}^{-1} P\left(Q_{1}\right) \cap u_{2}^{-1} P\left(Q_{2}\right)$. To finish the proof of the lemma remark that

$$
0 \geqslant L(p)=\tilde{L}(p)=\int_{\Pi} p \mathrm{~d} \mu>0,
$$

a contradiction.

A famous theorem of Kolmogorov, refined by Prokhorov, Bochner and other authors, asserts that a countable and directed projective system of probability measure spaces has a probability measure space as a projective limit, see for instance [1] Chapter 5, or [2] §4. A couple of applications of this result, following the lines of the preceding proof, can be stated as follows:

Theorem 2.3. Let $X=\operatorname{proj} \cdot \lim \left(X_{i}, f_{i j}\right)$ be a countable, directed projective limit of affine real varieties. Let $Q_{i} \subset$ $\mathbb{R}\left[X_{i}\right]$ be archimedean quadratic modules such that $f_{i j}^{*} Q_{i} \subseteq Q_{j}$. Let $p \in \sum_{i} f_{i}^{*} \mathbb{R}\left[X_{i}\right]$ be an element which is positive on the set $\bigcap_{i \in I} f_{i}^{-1} P\left(Q_{i}\right)$. Then $p \in \sum_{i} f_{i}^{*} Q_{i}$.

By definition, the above sums are algebraic, that is only finitely many terms in $p=\sum_{i} p_{i}, p_{i} \in f_{i}^{*} \mathbb{R}\left[X_{i}\right]$ are non-zero. When taking limits of elements in the algebraic sums above, we are led to the following variation:

Corollary 2.4. In the condition of the theorem, let $f \in\left[\sum_{i} f_{i}{ }^{*} \mathbb{R}\left[X_{i}\right]\right]^{-}$satisfy $f \geqslant 0$ on the compact set $\Pi=$ $\bigcap_{i \in I} f_{i}^{-1} P\left(Q_{i}\right)$. Then $f \in\left[\sum_{i} f_{i}^{*} Q_{i}\right]^{-}$where the closure in both cases is taken with respect to the uniform convergence on $\Pi$.

For the proof of the corollary, we again assume by contradiction that $f$ does not belong to the closed cone in the statement, and invoke a separation functional result (Minkowski in this case), providing a linear functional $L$ on $\left[\sum_{i} f_{i}^{*} \mathbb{R}\left[X_{i}\right]\right]^{-}$which satisfies

$$
L(f)<0 \leqslant L(g), \quad g \in\left[\sum_{i} f_{i}^{*} Q_{i}\right]^{-} .
$$


Kolmogorov's Theorem yields then a positive measure $\mu$ supported by $\Pi$, and representing $L$. Then

$$
0>L(f)=\int_{\Pi} f \mathrm{~d} \mu \geqslant 0,
$$

a contradiction.

We now apply Lemma 2.2 to the case of fibre products of affine spaces. Specifically, let $X_{1}=\mathbb{R}^{n_{1}} \times \mathbb{R}^{m}, X_{2}=\mathbb{R}^{m} \times$ $\mathbb{R}^{n_{2}}$ and $Y=\mathbb{R}^{m}$, while $f_{1}, f_{2}$ are the corresponding projection maps onto $Y$. Denote by $x_{1}, y, x_{2}$ the corresponding tuples of variables. Then one immediately identifies $Z=\mathbb{R}^{n_{1}} \times \mathbb{R}^{m} \times \mathbb{R}^{n_{2}}$, and Lemma 2.2 yields:

Corollary 2.5. Let $Q_{x_{1}, y}, Q_{y, x_{2}}$ be Archimedean quadratic modules in the respective sets of variables. Let $\Pi=$ $\left(P\left(Q_{x_{1}, y}\right) \times \mathbb{R}^{n_{2}}\right) \cap\left(\mathbb{R}^{n_{1}} \times P\left(Q_{y, x_{2}}\right)\right) \subset Z$. If a polynomial $p\left(x_{1}, y, x_{2}\right)=p_{1}\left(x_{1}, y\right)+p_{2}\left(y, x_{2}\right)$ is positive on $\Pi$, then $p \in Q_{x_{1}, y}+Q_{y, x_{2}}$.

\section{References}

[1] S. Bochner, Harmonic Analysis and the Theory of Probability, Univ. California Press, Berkeley, 1955.

[2] N. Bourbaki, Intégration, Chapitre IX, Hermann, Paris, 1969,

[3] D. Grimm, T. Netzer, M. Schweighofer, A note on the representation of positive polynomials with structured sparsity, Preprint, 2006.

[4] M. Kojima, M. Muramatsu, A note on sparse SOS and SDP relaxations for polynomial optimization problem over symmetric cones, Res. Report B-421, Math. Dept. Tokyo Inst. Techn., 2006.

[5] G. Köthe, Topological Vector Spaces. I, Springer, Berlin, 1969.

[6] S. Kuhlmann, M. Marshall, Positivity, sums of squares, and the multi-dimensional moment problem I, Trans. Amer. Math. Soc. 354 (2002) 4285-4301.

[7] S. Kuhlmann, M. Marshall, N. Schwartz, Positivity sums of squares, and the multi-dimensional moment problem II, Adv. Geom. 5 (2005) 583-607.

[8] J.B. Lasserre, Convergent semidefinite relaxations in polynomial optimization with sparsity, SIAM J. Optim. 17 (2006) $796-817$.

[9] J. Nie, J. Demmel, Sparse SOS relaxations for minimizing functions that are summation of small polynomials, Preprint http://arxiv.org.abs. math.OC/0606476.

[10] V. Powers, C. Scheiderer, The moment problem for non-compact semialgebraic sets, Adv. Geom. 1 (2001) 71-88.

[11] A. Prestel, C. Delzell, Positive Polynomials, Springer, Berlin, 2001.

[12] M. Putinar, Positive polynomials on compact semi-algebraic sets, Indiana Univ. Math. J. 42 (1993) 969-984.

[13] M. Putinar, C. Scheiderer, Multivariate moment problems: geometry and indeterminateness, Ann. Scuola Norm. Sup. Pisa Cl. Sci. (5) V (2006) 137-157.

[14] K. Schmüdgen, The $K$-moment problem for compact semi-algebraic sets, Math. Ann. 289 (1991) 203-206.

[15] H. Waki, S. Kim, M. Kojima, M. Muramatsu, Sums of squares and semidefinite programming relaxations for polynomial optimization problems with structured sparsity, SIAM J. Optim. 17 (2006) 218-242. 